\title{
The effects of acute consumption of a blackcurrant juice drink on markers of endothelial function as a risk factor for CVD
}

\author{
D. Alimbetov, Y. N. Jin, M. H. Gordon and J. A. Lovegrove \\ Hugh Sinclair Unit of Human Nutrition, Department of Food Biosciences, The University of Reading, Whiteknights, \\ PO Box 226, Reading, Berks RG6 6AP, UK
}

CVD is the major cause of death in most industrialised countries. Consumption of fruits and vegetables has been associated with a reduction in the risk of CVD and cancer, which may be at least partly due to the action of phytochemicals such as flavonoids and carotenoids $^{(1)}$. Flavonoids have antioxidant effects and are known to induce endothelium-dependent vasodilation by the nitric oxide guanosine cyclic monophosphate (NO-cGMP) pathway ${ }^{(2)}$.

The effect of acute consumption of $250 \mathrm{ml}$ of a drink containing $20 \%$ blackcurrant juice on vascular function, bioavailability and risk factors for CVD was investigated. The study was a double-blind, randomised controlled crossover dietary intervention study with a 4-week washout period. Test or control drink $(250 \mathrm{ml})$ was consumed on the study day by 20 volunteers who had fasted overnight and had followed a low-flavonoid diet for $3 \mathrm{~d}$ prior to the study day. Blood samples were taken at baseline and at 12 time points during the subsequent $8 \mathrm{~h}$. Urine samples were collected at baseline, at $2 \mathrm{~h}$ intervals during the study day, and a combined urine sample covering 8-24h was collected the following day.

The acute effect of consumption of a $20 \%$ blackcurrant juice drink on vascular reactivity was investigated by using Laser Doppler Imaging with iontophoresis (LDI). Measurements were taken at baseline and at $2 \mathrm{~h}$ after the consumption of test or control drink using acetylcholine and sodium nitroprusside to induce endothelium-dependent and -independent vasodilation, respectively. The vascular reactivity showed a tendency to fall after the consumption of control in response to both acetylcholine and sodium nitroprusside, whereas there was no reduction after the consumption of juice in response to acetylcholine. However, these effects on endothelium-dependent vasodilation were not statistically significant. There was no significant effect of treatment on total plasma nitrate and nitrite and no correlation between vascular reactivity and total plasma nitrate and nitrite concentrations. In conclusion, the consumption of the $20 \%$ blackcurrant juice did not result in any significant change in endothelial function.

The project was funded by GlaxoSmithKline.

1. Hertog MGL, Kromhout D, Aravanis C et al. (1995) Arch Intern Med 155, 381-386.

2. Stein Jamie H, Keevil JG, Wiebe DA et al. (1999) J Am Heart Assoc 100, 1050-1055. 